\title{
Evaluation of Beauveria sp strains, conidial concentration and immersion times on mortality rate of bovine tick (Boophilussp).
}

\author{
Oscar Miguel Domínguez-Galdámez ${ }^{1}$,María Ángela Oliva-Llaven ${ }^{1}$, Gabriela Aguilar-Tipacamú ${ }^{2}$, Paula Mendoza- \\ Nazar $^{1}$, Benigno Ruiz-Sesma ${ }^{1}$, Gerardo Uriel Bautista-Trujillo ${ }^{1}$, José Miguel Culebro-Ricaldi ${ }^{3}$, Federico Antonio \\ Gutiérrez-Miceli $^{3 *}$ \\ ${ }^{1}$ Universidad Autónoma de Chiapas, Facultad de Medicina Veterinaria y Zootecnia, ${ }^{2}$ Facultad de Ciencias Naturales, Universidad Autónoma de Querétaro, \\ ${ }^{3}$ Tecnológico Nacional de México. Instituto Tecnológico de Tuxtla-Gutiérrez, Laboratorio de Biotecnología Vegetal. Tuxtla-Gutiérrez, Chiapas, México.
}

\author{
ARTICLE INFO \\ Article history: \\ Received on: 04/04/2016 \\ Revised on: 28/04/2016 \\ Accepted on: 15/05/2016 \\ Available online: 26/08/2016 \\ Key words: \\ Bovine ticks, biological \\ control, in vitro bioassays, \\ native Beauveria $s p$ strains.
}

\begin{abstract}
Ticks (Boophilus sp) are one of the main problems that affect theperformance of bovine production in tropic and sub-tropical. Organophosphate acaricides are the usual tick control method,however the ecological damage and cross-resistanceto those acaricides isleading to the search for alternatives. Beauveria bassiana can be used as a biological control, however, laboratory bioassays with ticks showed mortality of 20 to $50 \%$ only. The aim of this investigation was to study the effect of five strains of Beauveria sp, two conidial concentrations and three immersion times on mortality rate of ticks. Five strains of Beauveria sp isolated in México were used and cultivated in liquid medium until $10^{7}$ and $10^{8}$ conidia/mL were obtained. For in vitro bioassays, ticks were collected from bovine, immersed in conidial suspensions for 20, 30 and 40 seconds. Mortality rate was recorded daily up to 10 days.Strain type was the main factor and 23-D Beauveriastrain hadthe highest value (100\%) of mortality rate after ten days of incubation with ticks. The best immersion time was $20 \mathrm{~s}$ and the bestconidial concentration was $10^{8}$ conidial $/ \mathrm{ml}$. In conclusion, native Beauveria sp strains could be an alternative viable for ticks control in bovine, however field test and Beauveria identification are necessary.
\end{abstract}

\section{INTRODUCTION}

Internal and external parasites of cattle remain one of the main causes of economic losses in Latin America and other livestock regions of the tropics and subtropics of the world [1]. Over the past four decades the development of miticides, insecticides and anthelmintics highly effective withbroad spectrum and residual power, has allowed the farmer to control these plagues. However, instead of solving the problem, they settled insecticides to kill both natural predators and pests [2]. The death of natural predators caused other insect plagues that used to be under control, increasing their populations significantly. Many species of insects considered plagues have developed resistance to one or more insecticides [3].The presence of ticks is one of the significant problems that affect performance in livestock production units [4]. However, the

\footnotetext{
* Corresponding Author

Federico Antonio Gutiérrez Miceli. Instituto Tecnológico de Tuxtla-

Gutiérrez, Carretera Panamericana km 1080, Tuxtla Gutiérrez,

Chiapas, México. E mail: fgmiceli@gmail.com
}

levels of resistance to two organophosphate acaricides, coumaphos and diazinon, in several Mexican strains of Boophilus microplus (Canestrini) were evaluated and data revealed a significant crossresistance pattern between those two acaricides [5]. Therefore the search for alternative control with an emphasis on natural controls and lesser ecological damage has been stimulated. An alternative is the use of entomopathogenic fungi (EF) such as Metarhizium, Beauveria, Paecilomyces, Verticillium, Fusarium and Rhizopus [6]. In particular, B. bassiana has been proved to be an efficient and environmentally friendly biocontrol agent against a variety of plagues $[7,8,9]$. Unlike other microorganisms, B. bassiana does not need to be ingested to exert their pathogenicity, and that penetrate through cuticle due to its ability to excrete enzymes (proteases, kinases, lipases, lipoxygenases) [10]. It has been reported that B. bassianacan be used for biological control of the cattle ticks, however, laboratory bioassays showed a mortality of only 20 to $50 \%$ of the ticks seven days after inoculation with $10^{7}$ Beauveria conidia/mL. Mortality 
values differed for each Beauveria strain tested [11]. Therefore; the aim of this investigation was to study the effect of five different strains of Beauveria sp, two conidial concentrations, three immersion times and ten incubation days on mortality rate of ticks (Boophilus microplus).

\section{MATERIALS AND METHODS}

Five strains of Beauveria sp were used. Bb-19 strain was donated from the Mycological collection of El Colegio de la Frontera Sur (ECOSUR), Chiapas, Mexico, Tapachula unit; while the 23D, 12.11, 12.15 and 12.05 strains were donated from the Mycological collection of the National Research Institute of Forestry, Agriculture and Livestock (INIFAP-General Teran), Nuevo Leon, Mexico.

Inoculation was made from a Petri dish containing pure strains of Beauveria sp. with 10 days of active growth in Agar potato medium. The preparation of liquid medium was done using $250 \mathrm{~mL}$ Erlenmeyer flasks with $50 \mathrm{~mL}$ of liquid medium containing minerals $\left(\mathrm{NaNO}_{3}, \mathrm{~K}_{2} \mathrm{HPO}_{4}, \mathrm{MgSO}_{4}, \mathrm{KCL}\right.$, and $\left.\mathrm{FeSO}_{4}\right)$, vegetable juice (celery, watercress, beets, spinach, tomatoes, lettuce, parsley and carrot), chitin $1 \%$ and Tween 80 , the flasks were autoclaved $\left(121^{\circ} \mathrm{C}, 15 \mathrm{lb} / \mathrm{inch}^{2}\right.$ for $\left.15 \mathrm{~min}\right)$. Inoculation with B. sp was performed in laminar flow chamber previously disinfected with $70 \%$ alcohol. Flasks were kept under constant stirring at $180 \mathrm{rpm}$ for 3 days at room temperature, where a concentration of $10^{7}$ and $10^{8}$ conidia / $\mathrm{mL}$ were obtained. The counting of conidial was done according to Cañedo[12]. Briefly, a sample of liquid medium was taken with a pipette; a small drop was placed on each of the deposits of the Neubauer chamber. The concentration of conidial was calculated as follows:

$$
N C=\left(\frac{S C}{5}\right) * 25 * 50,000
$$

Where:

$\mathrm{NC}=$ number of conidial per milliliter of suspension.

$\mathrm{SC}=\mathrm{Sum}$ of conidia contained in the five pictures from the Neubauer chamber.

\subsection{Collection of ticks in animals.}

Ticks were collected directly from bovine animals, making a previous interview of the production unit in order to obtain information regarding the background to the use of external antiparasitic. Subsequently we carried out the collection of ticks with a simple random method. We randomly sampled 30 animals and we collected 30 adult ticks for identification post. The selected animals were handled individually, and subsequently underwent a rigorous visual physical examination and palpation of the skin, to detect ticks at any stage. The examination was performed systematically in pre anatomical regions: Pavilion ears, head, neck, back, earlier train, underarms, chest, belly, udder, scrotum, flanks, groin, hindquarter, perineum and tail. Ticks were deposited in test tubes with cotton plugs. Ticks were transported to laboratory of Parasitology in the Faculty of Veterinary Medicine at the Autonomous University of Chiapas (FMVZ -UNACH). Adult ticks were placed in glass vials with cotton plugs to allow breathing, incubated at $27^{\circ} \pm 2^{\circ} \mathrm{C}$ and with $80-85 \%$ relative humidity $(\mathrm{RH})$ to stimulate oviposition. Eggs were placed in glass vials covered with filter paper under the same conditions of temperature and relative humidity. Larvae 14 days old were used in bioassays for the evaluation of pathogenicity of Beauveria sp. For the pathogenicity evaluation of the isolated strains of the fungus $B$. $s p$ on populations of ticks, a bioassay was installed under controlled laboratory conditions (temperature and relative humidity) using a factorial design $5 \times 3 \times 2$ completely randomized with 30 treatments and four replications for each one. To calculate the mortality rate, 20 larvae of ticks were immersed in Beauveria. sp suspensions with $10^{7}$ and $10^{8}$ conidia/ml for 20,30 and 40 seconds and then placed in a paper towel to absorb excess of liquid medium. The control group was treated with sterile water and $1 \%$ Tween 80 in place of the suspension of conidia.

\subsection{Statistical analysis}

The effect of treatment was determined by analysis of variance with a significance level of 5\% and the mean test was conducted by Media Significant Difference test. The Statgraphic Plus (Statgraphics Centurion XV, 2007) was used for the regression analysis of the experimental data.

\section{RESULTS AND DISCUSSIONS}

In Table 1 are presented the different treatments according to the experimental design factorial complete $5 \times 3 \times 2$, results of mortality percentage of ticks isolated from bovine exposed to different Beauveria sp strains, immersion times and conidial concentrations. Mortality percentage of Boophilus sp varied from 60 to 100 after ten days of incubation time (Table 1).

ANOVA for main effects and interactions of strain, conidial concentration and immersion time Bauvaeria sp on mortality percentage of ticks are presented in Table 2. Straintype of Beauveria sp and interactions had a significant effect on mortality rate of tick. In relation with the strain type, the 23-D strain had the highest value (100\%), whereas the lowest value $(82 \%)$ of mortality rate of tick (Boophilus sp) was obtained with 1211 strain after ten days of incubation (Table 3). The effects of Bauvaeria sp strain 23-D on mortality percentage of tick (Boophilus sp) started after two day of incubation and gradually increased until it reaches $100 \%$ of mortality (Figure 1).

Strain type of Beauveria sp and interaction between strain type, immersion time and conidial concentration had a significant effect on mortality rate of tick. 23-D strain had the highest value (100\%), whereas the lowest value (82\%) of mortality rate of tick (Boophilus sp) was obtained with 1211 strain. These results could be explained for differences among enzyme activities in Beauveria strain type and it is necessary the characterization of certain enzymes such as phosphoenolpyruvate carboxykinase, isocitratelyase and pyruvate carboxylasein each strain. These enzymes have been recognized as vital components of several biochemical reactions in other fungi and bacteria [3]. 
Table 1: Experimental design factorial complete $5 \times 3 \times 2$ and results of mortality percentage of ticks isolated from bovine exposed to different Beauveria sp strains, immersion times and conidial concentrations. Evaluation was done after ten days of incubation days and results are media of five repetitions.

\begin{tabular}{|c|c|c|c|c|}
\hline Treatment & Strain & Immersion time & Conidial concentration & Mortality \\
\hline & & $-\mathrm{s}-$ & -Conidial/ml- & $-\%-$ \\
\hline 1 & 19 & 20 & 7 & 80 \\
\hline 2 & 19 & 20 & 8 & 90 \\
\hline 3 & 19 & 30 & 7 & 80 \\
\hline 4 & 19 & 30 & 8 & 90 \\
\hline 5 & 19 & 40 & 7 & 90 \\
\hline 6 & 19 & 40 & 8 & 90 \\
\hline 7 & 1205 & 20 & 7 & 90 \\
\hline 8 & 1205 & 20 & 8 & 90 \\
\hline 9 & 1205 & 30 & 7 & 95 \\
\hline 10 & 1205 & 30 & 8 & 95 \\
\hline 11 & 1205 & 40 & 7 & 95 \\
\hline 12 & 1205 & 40 & 8 & 90 \\
\hline 13 & 1211 & 20 & 7 & 90 \\
\hline 14 & 1211 & 20 & 8 & 80 \\
\hline 15 & 1211 & 30 & 7 & 80 \\
\hline 16 & 1211 & 30 & 8 & 80 \\
\hline 17 & 1211 & 40 & 7 & 100 \\
\hline 18 & 1211 & 40 & 8 & 60 \\
\hline 19 & 1215 & 20 & 7 & 90 \\
\hline 20 & 1215 & 20 & 8 & 100 \\
\hline 21 & 1215 & 30 & 7 & 90 \\
\hline 22 & 1215 & 30 & 8 & 100 \\
\hline 23 & 1215 & 40 & 7 & 90 \\
\hline 24 & 1215 & 40 & 8 & 100 \\
\hline 25 & $23 \mathrm{D}$ & 20 & 7 & 100 \\
\hline 26 & $23 \mathrm{D}$ & 20 & 8 & 100 \\
\hline 27 & $23 \mathrm{D}$ & 30 & 7 & 100 \\
\hline 28 & 23D & 30 & 8 & 100 \\
\hline 29 & $23 \mathrm{D}$ & 40 & 7 & 100 \\
\hline 30 & $23 \mathrm{D}$ & 40 & 8 & 100 \\
\hline control & & & & 0 \\
\hline
\end{tabular}

Table 2: ANOVA for main effects and interactions of strain, conidial concentration and immersion time Bauvaeriasp on mortality percentage of tick (Boophilus $s p)$. Data used were obtained after ten days of incubation.

\begin{tabular}{lcccc}
\hline Source & Square sum & Df & Mean square & F- reason \\
\hline Main effect & & & & P- value \\
A:strain & 6151.67 & 4 & 1537.92 & 128.89 \\
B:Inmmersion time & 16.0 & 2 & 8.0 & 0.0000 \\
C:Conidial concentration & 0 & 1 & 0 & 0.5133 \\
Interactions & & & 0.00 & 1.0000 \\
AB & 337.333 & 8 & 42.1667 & 3.53 \\
AC & 3201.67 & 4 & 800.417 & 0.0010 \\
BC & 916.0 & 2 & 458.0 & 0.0000 \\
Residual & 1527.33 & 128 & 11.9323 & 0.0000 \\
TOTAL (corrected) & 12150.0 & 149 & & \\
\hline
\end{tabular}

All-F reasons are based on the mean square of residual error

The effects of Bauvaeria sp strain 23-D in mortality percentage of tick (Boophilus sp) started after two day of incubation and gradually increased until it reaches $100 \%$ of mortality. This kinetic pattern could be explained because Beauveria bassiana infects insects by direct cuticle penetration rather than by ingestion or through a wound like viruses or bacteria [13]. The infection process consists in three stages: 1) attach to the cuticle, 2) penetrate the cuticle, and 3) proliferate in the haemocoel and kill the host. The whole process is rather complex and multiple host factors and fungal toxins could be involved in the process [14]. Although insect resistance to $B$. bassiana has not yet been reported, some disadvantages impede the wide use of fungal biological agents. For example, they are not as fast acting as chemical insecticides and their efficacy varies with field conditions. Successful application of B. bassiana needs favorable environmental conditions such as high humidity and medium temperature. Recently Xiao [10] sequenced the genome of B. bassiana and a phylogenomic analysis confirmed that ascomycete entomopathogenicity is polyphyletic, but also revealed convergent evolution to insect pathogenicity. B. bassianais having more bacterial-like toxins (suggesting an unsuspected potential for oral toxicity) and effector-type proteins. A high throughput RNAseq transcriptomic analysis revealed that $B$. bassiana could sense and adapt to different environmental niches by activating welldefined gene sets [10].

In our study, the better strain was the 23-D, donated for Mycological collection of the National Research Institute of Forestry, Agriculture and Livestock (INIFAP) Mexico. It will be necessary to characterize this strain both genetically and metabolically. 


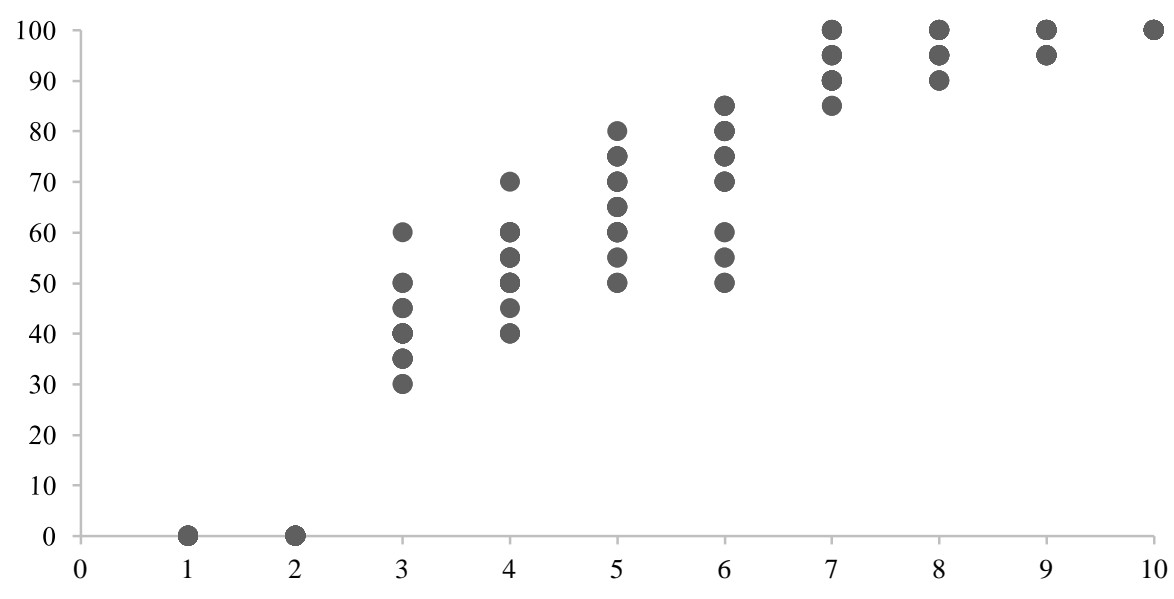

Incubation time (days)

Fig 1: Effects of Bauvaeriasp strain 23-D on mortality percentage of tick (Boophilus sp).

Table 3: Evaluation of strain, conidial concentration and immersion time of Bauvaeriasp on mortality percentage of tick (Boophilus sp).

\begin{tabular}{|c|c|c|c|c|c|c|}
\hline & & & Standard & Homogeneous & Lower & Upper \\
\hline Factors & observations & Media & Error & group & limit & Limit \\
\hline \multicolumn{7}{|l|}{ strain } \\
\hline 1205 & 30 & 91.8 & 0.63 & $\mathrm{c}$ & 90.58 & 93.08 \\
\hline 1211 & 30 & 81.7 & 0.63 & $\mathrm{e}$ & 80.41 & 82.91 \\
\hline 1215 & 30 & 95.0 & 0.63 & b & 93.75 & 96.24 \\
\hline 19 & 30 & 86.5 & 0.63 & $\mathrm{~d}$ & 85.25 & 87.74 \\
\hline 23D & 30 & 100.0 & 0.63 & $\mathrm{a}$ & 98.75 & 101.28 \\
\hline $\operatorname{LSD}(0.05)$ & & 1.76 & & & & \\
\hline \multicolumn{7}{|l|}{ immersion time } \\
\hline 20 & 50 & 91.0 & 0.49 & $\mathrm{a}$ & 90.03 & 91.96 \\
\hline 30 & 50 & 90.6 & 0.49 & $\mathrm{a}$ & 89.63 & 91.56 \\
\hline 40 & 50 & 91.4 & 0.49 & a & 90.43 & 92.36 \\
\hline LSD $(0.05)$ & & 1.37 & & & & \\
\hline \multicolumn{7}{|l|}{ conidial concentration } \\
\hline $1 \times 10^{7}$ & 75 & 91.0 & 0.39 & a & 90.2108 & 91.7892 \\
\hline $1 \times 10^{8}$ & 75 & 91.0 & 0.39 & $\mathrm{a}$ & 90.2108 & 91.7892 \\
\hline $\operatorname{LSD}(0.05)$ & & 1.12 & & & & \\
\hline
\end{tabular}

${ }^{a}$ Values are media calculated with all incubated days and two conidial concentrations. ${ }^{b}$ Values are media calculated with all strains and all incubation days.

${ }^{\mathrm{c}}$ Values are media calculated with all strain and two conidial concentrations.

\section{CONCLUSION}

Beauveria sp can be used for controlling ticks in bovine, however the effects depend heavily on the strain used rather than the conditions of application of the fungus. Additional field tests and Beauveria sp identification are necessary to obtain data that can support the commercial application of this method of biological control of ticks

\section{REFERENCES}

1. Torgerson PR, Macpherson, CNL. The socioeconomic burden of parasitic zoonoses: Global trends. Veterinary Parasitology.2011; 182: 79-95. doi: http://dx.doi.org/10.1016/j.vetpar.2011.07.017

2. Perry AS, Perry I, Yamamoto I,Ishaaya R, Perry Y. Environment: Retrospects and Prospects. Springer Science \& Business Media, $14 / 03 / 2013-261 p$.

3. Xia J, Zhang C-R, Zhang S, Li F-F, Feng M-G. Analysis of whitefly transcriptional responses to Beauveria bassiana infection reveals new insights into insect-fungus interactions. PLoS ONE. 2013;8: e68185. doi:10.1371/journal.pone.0068185

4. Muchenje Dzama K, Chimonyo M, Raats JG, Strydom PE. Tick susceptibility and its effects on growth performance and carcass characteristics of Nguni, Bonsmara and Angus steers raised on natural pasture. Animal Cambridge Journals. 2008;2:288-298. DOI: 10.1017/S1751731107001036

5. Andrew YL, R DaveyRJ, Miller George JE. Resistance to coumaphos and diazinon in Boophilus microplus (Acari: Ixodidae) and evidence for the involvement of an oxidative detoxification mechanism. J. Med. Entomol. 2003;40: 482-490

6. Sujeetha JA, RP, Sahayaraj K. Role of entomopathogenic fungus in pest management. In K. Sahayaraj (Ed.), Basic and Applied Aspects of Biopesticides. New Delhi: Springer India. 2014; pp. 31-46.

7. Daniel C, Wyss E. Field applications of Beauveria bassiana to control the European cherry fruit fly Rhagoletiscerasi. J Appl Entomol. 2010; 134: 675-681.

8. Torrado-Leon E, Montoya-Lerma J, Valencia-Pizo E.Sublethal effects of Beauveria bassiana (Balsamo) Vuillemin (Deuteromycotina: Hyphomycetes) on the whitefly Bemisiatabaci (Gennadius)(Hemiptera: Aleyrodidae) under laboratory conditions. Mycopathologia. 2006; 162: 411-419. 
9. Wraight S, Carruthers R, Jaronski S, Bradley C, Garza C. Evaluation of the entomopathogenic fungi Beauveria bassiana and Paecilomycesfumosoroseus for microbial control of the silverleaf whitefly, Bemisiaargentifolii. Biol Control. 2000; 17: 203-217

10. Xiao G, Ying S-H, Zheng P, Wang Z-L, Zhang S, Xie X-Q, Feng MG. Genomic perspectives on the evolution of fungal entomopathogenicity in Beauveria bassiana. Scientific Reports. 2012;2,483.doi:10.1038/srep00483.http://www.nature.com/articles/sr ep00483\#supplementary-information

11. Campos RA, JT Boldo, IC Pimentel, V Dalfovo, WL Araújo, JL Azevedo, MH Vainstein,Barros NM. Endophytic and entomopathogenic strains of Beauveria $s p$ to control the bovine tick Rhipicephalus (Boophilus) microplus Genetics and Molecular Research. 2010; 9: 1421-1430.

12. Cañedo V, Ames T. Manual de Laboratorio para el Manejo de Hongos Entomopatógenos. Lima, Perú; Centro Internacional de la Papa (CIP). 2004. Lima, Perú, 62 p.
13. Faria M, Wraight SP. Biological control of Bemisiatabaci with fungi. Crop Prot. 2001; 20: 767-778.

14. Toledo AV, de Remes Lenicov AMM, Lastra CCL.Histopathology caused by the entomopathogenic fungi, Beauveria bassiana and Metarhiziumanisopliae, in the adult planthopper, Peregrinusmaidis, a maize virus vector. J Insect Sci. 2010; 10: 35.

\section{How to cite this article:}

Domínguez-Galdámez OM, Oliva-Llaven MA, Aguilar-Tipacamú G, Mendoza-Nazar P, Ruiz-Sesma B, Bautista-Trujillo GU, Culebro-Ricaldi M, Gutiérrez-Miceli FA. Evaluation of Beauveria $s p$ strains, conidial concentration and immersion times on mortality rate of bovine tick (Boophilussp). J App Biol Biotech. 2016; 4 (04): 064-068. DOI: 10.7324/JABB.2016.40407 